\title{
Palliative Oral Healthcare for Patients with Terminal Stages of Malignancy
}

\author{
Sheikh Abrar and Pavan Manohar Patil* \\ Department of Oral and Maxillofacial Surgery, School of Dental Sciences, Uttar Pradesh, India \\ Department of Oral and Maxillofacial Surgery, Professor and Head of Department, School of Dental Sciences, Uttar Pradesh, \\ India \\ *Corresponding author: Pavan Manohar Patil, Department of Oral and Maxillofacial Surgery, Professor and Head of Department, \\ School of Dental Sciences, Uttar Pradesh, India
}

\begin{tabular}{|c|c|}
\hline ARTICLE INFO & ABSTRACT \\
\hline Received: 慧 September 29, 2020 & Citation: Sheikh Abrar and Pavan Manohar Patil. Palliative Oral Healthcare for Patien \\
\hline Published: 幽 October 09, 2020 & $\begin{array}{l}\text { with Terminal Stages of Malignancy. Biomed J Sci \& Tech Res 31(1)-2020. BJSTR. } \\
\text { MS.ID.005046. }\end{array}$ \\
\hline
\end{tabular}

\section{Introduction}

Oral symptoms typically increase as end of life approaches in patients on palliative care for terminal stages of malignancy. Hence, the focus of oral healthcare providers is to manage symptoms and maximize quality of life during the end-of-life transition. The American Society of Clinical Oncology (ASCO), in a recently updated clinical practice guideline, strongly recommends that palliative care be provided to all patients with advanced cancer early in the disease course, at the same time as active treatment [1]. Wiseman [2] advocated that symptom relief was the primary principle of palliative care dentistry, which focused on treatment of terminally ill patients in whom the oral cavity was affected directly or indirectly by the illness. Care for the oral cavity problems assumes great importance since it provides an important route for nutrition, medications, speech and affection by kissing. Medical management of palliative care patients with chemotherapy/radiotherapy or both, often lead to development of oral complications among these patients. A sneak preview into the oral cavity problems in palliative care patients can be gauged by the report of National Cancer Institute at the National Institutes of Health, United States of America, which indicated that $80 \%$ of patients receiving myeloablative chemotherapy will develop oral complications, and oral mucositis or taste disturbances were associated with the use of palliative drugs such as bisphosphonates and analgesics [3].

The pain and suffering of palliative patients with oral conditions may be minimized by early diagnosis and treatment of oral conditions [4]. However, there is widespread under reporting and underestimation of oral health care problems in palliative care patients. $40 \%$ of palliative patients are unable to communicate their oral health needs or may not complain of discomfort in their oral cavity, erroneously believing that such conditions are an inevitable effect of their palliative medical therapy [5].

\section{Oral Effects of Palliative Cancer Care}

The oral problems faced by palliative cancer care patients are listed in Table 1. These problems will be discussed in terms of their management strategies in the following sections.

Table 1: Oral Problems Faced by Palliative Care Patients.

\begin{tabular}{|c|}
\hline Manifestation \\
\hline Mucositis/Stomatitis \\
\hline Hyposalivation/Xerostomia \\
\hline Nausea and vomiting (tooth erosion and sensitivity) \\
\hline Orofacial burning/pain \\
\hline Fungal infections \\
\hline Dental caries \\
\hline Periodontal disease \\
\hline Taste disturbances \\
\hline Dysphagia \\
\hline Tongue coating/inflammation \\
\hline Gingival inflammation \\
\hline
\end{tabular}




\begin{tabular}{|c|}
\hline Mucosal ulceration \\
\hline Dysarthria \\
\hline Halitosis \\
\hline Coughing and problems in denture use \\
\hline Bleeding spots \\
\hline Cold sores \\
\hline Leukoplakia \\
\hline
\end{tabular}

\section{Oral Mucositis / Stomatitis}

Table 2: Grading Indices for Oral Mucositis/Stomatitis.

\author{
World Health Organization (WHO) Grading of Mucositis \\ Grade 0 - no signs and symptoms \\ Grade 1 - painless ulcers, edema, or mild soreness \\ Grade 2 - painful erythema, edema, or ulcers but able to eat \\ Grade 3 - painful erythema, edema, or ulcers but unable eat \\ Grade 4 - requirement for parenteral or enteral support
}

National Cancer Institute Common Toxicity Criteria for Grading of Stomatitis

Grade 1 - Painless ulcers, erythema, or mild soreness

Grade 2 - painful erythema, edema, and ulcer but is able to eat

Grade 3 - inability to eat

Grade 4 - requiring parenteral or enteral support

Radiation Therapy Oncology Oral Mucositis Grading System

1 - erythematous sores

2 - patchy mucositis $(<1 / 2$ mucosa)

3 - fibrinous mucositis ( $>1 / 2$ mucosa)

4 - hemorrhage and necrosis

The oral cavity is affected by chemotherapy and radiotherapy which are used in the treatment of cancer patients and sometimes extended into the palliative period to reduce pain and improve function [6]. Tissues of the oral cavity that exhibit high mitotic turnover are affected by chemotherapy, leading to atrophy of the tissues. Radiotherapy brings about sclerosis of the small vessels which vascularise the oral tissues [7]. Indexes used to grade the severity of oral mucositis (OM) are outlined in Table 2 [6]. Pathophysiology involved in the development of OM was classically proposed in a five phase model by [8] in 2008. The initiation phase involves direct DNA damage from radio or chemotherapy to basal epithelial, submucosal, and endothelial cells. These damaged cells in turn release mediators that bind to specific receptors and lead to inflammation and toxicity [9]. As a result of this damage, oxidative stress forms reactive oxygen species that damage cell membranes, stimulate macrophages, and trigger molecules that activate transcription factors, including nuclear factor (NF)- $\kappa$ B which is considered to be the gatekeeper for inflammatory pathways involved in mucositis [7]. Its activation is associated with increased amounts of proinflammatory cytokines such as tumor necrosis factor (TNF)- $\alpha$, interleukin (IL)-6, and IL-1 $\beta$. It also uppregulates the enzyme cyclo-oxygenase-2 (COX-2) in submucosal fibroblasts and endothelial cells [7]. This is followed by the ulcerative phase which is characterized by loss of mucosal integrity and microbiological colonization by oral bacteria. The final phase is that of ulcer healing, characterized by epithelial proliferation (usually coexistent with hematopoietic recovery), local microbial reestablishment and absence of wound healing inhibiting factors such as infection and mechanical irritation [7].

Oral mucositis management is aimed at symptom management and prevention of complications, which may encompass strategies such as pain control, nutritional support and prophylaxis/treatment of secondary infections. Preventive measures could be started if the $\mathrm{OM}$ is undeveloped but patient is undergoing treatment which has a high propensity to cause OM. However, once OM develops, therapy must be instituted. WHO grade 1 and 2 mucositis must be treated with maintenance of oral hygiene, saline rinses, removal of dentures if they are irritating, closely monitoring nutritional status, reference to dietician if eating and drinking are affected, simple analgesia in the form of soluble paracetamol $1 \mathrm{~g}$ four times daily as mouthwash [10]. Non steroidal anti-inflammatory drugs (NSAIDs) are contraindicated due to the risk of bleeding and renal impairment10. Benzydamine hydrochloride $0.15 \%$ oral solution may be rinsed every 1.5 to 3 hours, as required. Increasing folinic acid rescue for methotrexate induced mucositis may be considered. Antibiotics are prescribed when there is evidence of oral infection. Consider use of Caphosol $^{\circledast}$ (EUSA Pharma,4-10 times a day) to prevent grade 1 and $2 \mathrm{OM}$ becoming more severe. WHO grade 3 and $4 \mathrm{OM}$ is treated with the use of stronger analgesia, including oxycodone hydrochloride $5 \%$ oral solution, morphine sulfa pentahydrate $10 \mathrm{mg}$ or morphine oral solution $10 \mathrm{mg} / 5 \mathrm{ml}$ to alleviate pain. If the pain continues, consider using further opioid analgesia, such as fentanyl patches, patient-controlled analgesia or a syringe driver [10].

Laxatives are prescribed to prevent constipation and associated nausea. Consideration must be given to applying a coating protectant such as Gelclair $^{\circledR}$, Mugard $^{\circledR}$, Episil $^{\circledR}$ which is rinsed around the mouth to form a protective layer over the sore areas, applied d 1 hour before eating [10]. Chlorhexidine mouthwashes are contraindicated in any patient who has or is recovering from cytotoxic induced mucositis, as they tend to inhibit mucosal regrowth [10]. Recently, cryotherapy has been establisged as an effective treatment modality which is easy to use, cost effective and efficient [11]. Intraoral photobiomodulation using low level laser light is also producing promising results [12].

\section{Dysphagia}

Dysphagia may occur in either of its two phases; the oropharyngeal phase or the esophageal phase. The oropharyngeal phase may be affected by poor dentition, inadequate saliva production, sufficient muscular function, obstructed oral pathway, 
or pain from ulcers, herpes, or fungi [13]. Inability to masticate foods may result from tooth loss, ill-fitting dentures, dental caries, or advanced periodontal disease. Pain and function must be evaluated by the palliative care dentist. Dental caries and periodontal disease must be treated and prosthetic replacement of teeth to restore oral function should be based on prognostic longevity of the patient. Most palliative care patients may not be a good candidates for crowns or implants but may benefit from removable partial dentures [13].

\section{Nutritional Support}

It is estimated that $70 \%$ of terminally ill patients suffer from anorexia (lack or loss of appetite for food) [14]. Reasons include interference in mastication, depression, pain, stomatitis, dysphagia, nausea, depression and effect of medications used for palliative cancer therapy [15]. Nonpharmacologic methods to combat anorexia may include provision of more frequent, small highenergy meals, where the meal is made to look as appetizing as possible using colorful foods arranged in a visually pleasing way [15]. Preparing foods that the patient relishes is another way. Nutritional supplements and dietary counselling may assist in nutrition enhancement. Pharmacologic agents to stimulate the appetite include megestrol acetate, cannabinoids, progestins, anamorelin and corticosteroids [14]. These should be prescribed by the physician. Additionally, as the patient's mouth may be dry, moist and soft meals are recommended so that the patient may swallow it easily. High calorie shakes promote nutrition, sooth the mucosa and can be appetizing for the patient [15]. Use of food seasonings however may irritate the mucosa and must be checked for individual patient tolerance. Enteral and parenteral nutrtion are the last resort in palliative care patients. The National Comprehensive Cancer Network issued guidelines for Palliative Care in 2017 wherein it recommended that enteral and parenteral feeding be considered only in patients who had a prognosis for life of longer than weeks rather than days [16].

\section{Nausea and Vomiting}

Chemotherapy commonly results in nausea and vomiting as a side effect of chemotherapeutic drugs. Additional causes may be peptic ulcer disease, malignancy associated gastroparesis, metabolic disturbances, and opiod use. Chronic vomiting is of importance to the oral healthcare provider as the acid content of vomit can erode tooth enamel, cause tooth sensitivity and increases the severity of mucositis and stomatitis [15]. The use of fluoride rinses and fluoride varnish applications can counteract the erosive effect of chronic vomiting. The Multinational Association for Supportive care in cancer/European society for medical oncology MASCC/ESMO consensus recommendations 2016 for management of nausea and vomiting in advanced cancer advise the use of metoclopramide (titrated to effect) as the first choice drug, with alternative options being haloperidol, levomepromazine, or olanzapine [17]. Octreotide is recommended in cases of bowel obstruction, given alongside an antiemetic (haloperidol) or when inappropriate, to use an anticholinergic antisecretory agent such as scopolamine butylbromide or glycopyrronium bromide [17]. For opioid-induced nausea and vomiting, no recommendation could be made. However, it was opined that opioid rotation and route switching may be effective approaches [17]. Fluid replacement is advisable through parenteral routes such as intravenous or subcutaneous routes. Oral fluids must be started as soon as the patient is able to tolerate.

Nausea can make denture wearing difficult, which hinders mastication and more importantly, compromise their quality of life by affecting their social interactions [15]. Oral hygiene must be instituted as a strict regimen, taking care to prevent triggering a gagging reflex with the use of a smaller toothbrush (child size) if necessary.

\section{Xerostomia/Dry Mouth}

Xerostomia or dry mouth is believed to be present if the patient states that his/her mouth is dry, although, the oral mucosa may appear to be moist. Salivary gland hypofunction on the other hand is defined by a quantitative salivary flow rate of $<0.7 \mathrm{ml} / \mathrm{min}$ [18]. Xerostomia is commonly encountered in palliative care patients with medication use the most common cause, notably analgesics, antidepressants, diuretics and antiemetics. The use of alcohol mouthwashes, and caffeinated beverages may just aggravate the condition15. The effects of xerostomia are observed in the quality of life of the patient as it affects their ability to eat, communicate, and interact socially [19]. Dental caries is significantly higher in xerostomic patients [20]. Additionally xerostomic patients prefer to eat foods that are softer and cariogenic [21]. The palliative oral healthcare provider must strive keep the mouth moist. Measures to ensure a moist mucosa by salivation is hydration, humidifying the patient's room and use of artificial saliva agents. Hydration of the oral mucosa can be achieved with the use of a water soluble lubricant such as 'Oral Balance gel' (lactoperoxidase, lysozyme, glucose oxidase, lactoferrin) or mucin-containing 'AS Saliva Orthana' [22]. Nursing assistants may apply the gel uniformly all around the oral cavity using a foam brush. Petroleum based products such as vaseline are not recommended as they are unpalatable, anhydrous and hydroscopic, thereby absorbing water from the tissues. Salivary elimination of bacteria from the oral cavity may also be impeded by their ability to occlude harmful bacteria [22]. There is a potential combustible threat from such products in patients on oxygen support.

Hydration may be achieved with simple measures such as frequent sips or sprays of cold water, sucking ice cubes or chewing sugar-free gum. Alchohol containing mouth rinses are not recommended as they tend to further desiccate the mouth [22]. Alcohol-free rinses should be used before meals to improve 
swallowing. Cholinergic-mimetic drugs such as pilocarpine (5mg thrice a day) or cevimeline in palliative care have been recommended as the first line therapy in head and neck cancer survivors with radiotherapy-induced xerostomia and hyposalivation [23]. However, in chemotherapy induced xerostomia, these drugs do not have the same level of evidence. Malic acid, vitamin C and citric acids used in topical application form can stimulate saliva. However, their low $\mathrm{pH}$ predisposes teeth to demineralization [22]. Newer methods to combat xerostomia, especially in the post radiotherapy palliative care patient include acupuncture-like transcutaneous electrical nerve stimulation (ALTENS) [24] and acupuncture alone [25]. The advantage of both are that they can be provided to the patient at home and therefore no hospital visit is required.

\section{Candidiasis}

Also popularly known as oral thrush, it affects $70-85 \%$ of palliative care patients [22]. Factors that predispose patients include lack of oral hygiene, hyposalivation/xerostomia, anemia, immunosuppression, long term corticosteroid use, use of broad spectrum antibiotics, nutritional deficiencies, diabetes mellitus and denture wearing. Clinically, candidiasis may be of pseudomembranous, atrophic or hyperplastic type. Candidiasis may be treated by a combination of topical and systemic medications. Topical medications include Nystatin suspension (swish 3-5 times daily), sugarless fruit juice containing frozen nystatin suspension, nystatin vaginal suppository, clotrimazole vaginal suppository, clotrimazole troche, clotrimazole vaginal cream 1\% (denture application for a week) [22]. Systemic medications include options such as Ketoconazole (200-400 mg orally for 1-2 weeks), Fluconazole (100-200 mg on day 1, then $50-100 \mathrm{mg} /$ day orally for 1-2 weeks), Itraconazole (100-200 mg/day orally for 1-2 weeks) and Amphotericin B (0.25-1.5 mg/kg a day intravenously) in resistant cases [22]. Recently, a single dose regimen of Fluconazole $150 \mathrm{mg}$ has been reported to be effective in treatment of oral thrush [26].

Denture care is vital in preventing denture-related candidiasis. Dentures should be stored overnight in a dedicated vessel in solutions of water, $0.12 \%$ chlorhexidine solution, Listerine solution or $100000 \mathrm{IU}$ of nystatin suspension [22]. Soaking the denture in bleach $(15 \mathrm{~mL})$ and water $(250 \mathrm{~mL})$ for 30 minutes can assist in getting rid of unpleasant odour from the denture. However. the metal part of partial dentures may fatigue on immersion in bleach solution [22]. Benzalkonium chloride (1:750) can also be used for denture immersion for 30 minutes. Boiling the denture may distort the denture base, however, disinfection may be achieved by microwaving it in water at high power for 5 minutes. Repeated microwaving, however, is to be cautioned against as this can result in hardening of soft denture linings [22].

\section{Orofacial Pain}

The Indian society for study of pain (ISSP) Cancer Pain Special Interest Group guidelines (2019) [27] have provided clinical answers for palliative care pain management. ISSP recommends that in ambulatory palliative care patients, the stepladder analgesic model be used for cancer pain management. Patients' "level" of pain (mild, moderate, or severe) is determined through numerical rating scale for pain. Mild pain step 1: is treated using nonopioid analgesics (NSAIDs or acetaminophen), moderate pain step 2: is managed with a weak opioid analgesic, alone or in combination with a nonopioid or adjuvant analgesic) and severe pain step 3: is managed with a strong opioid analgesic, alone or in combination with a nonopioid or adjuvant analgesic) [27].

Some patients with poorly controlled cancer pain in ambulatory and home care require admission for acute inpatient pain management. Methods used to treat such individuals include rapid oral opioid titration along with adjuvants, correcting the treatable cause of pain using palliative radiotherapy, physiotherapy, or by correcting hypercalcemia, etc. [27]. Acute pain crisis is treated with admistration of intravenous opioid, followed by opioid infusion for maintenance therapy. Oral and transdermal preparations are substituted once stable pain relief is achieved. Neuromodulation is attempted using intravenous lignocaine or ketamine boluses, followed by maintenance dose with these drugs in cases with neuropathic pain crisis [27]. A consensus was published regarded use of methadone (opiod) in end-of-life cancer patients by the Hospice and Palliative Care experts group in 2019 [28]. Methadone therapy is indicated in cases of moderate to severe pain (especially as a second line opioid choice), pain not controlled by other opioids, allergy to morphine, significantly impaired renal function, when a long-acting opioid in oral solution form is needed, high opioid tolerance, opioid adverse effects from other opioids, patients with dysphagia/ inability to swallow, or feeding tube placement [28].

\section{Home Oral Care}

The importance of oral hygiene maintenance and care is frequently overlooked in terminally ill patients, especially if it is being carried out by a caregiver, until the person's quality of life is compromised by their oral symptoms [29]. Therefore, oral health maintenance through oral hygiene measures are vital in palliative oral care. Measures to be taken are enlisted in Table 3 [30-32]. 
Table 3: Oral Hygiene Maintenance Strategies in Palliative Care.

\begin{tabular}{|l|}
\hline 1. Encouragement and reinforcement of good oral hygiene maintenance \\
\hline 2. Tooth brushing twice daily and after meals with a soft to medium toothbrush, \\
\hline preferably with a fluoride-containing toothpaste \\
\hline 3. Change the toothbrush in case an oral infection develops \\
\hline 4. Toothpaste with a high content of fluoride (over $1450 \mathrm{ppm}$ ) may be required in certain individuals to protect the teeth \\
\hline 5. After brushing, toothpaste should not remain in the mouth but must be rinsed out with water \\
\hline 6. Brushing difficulties can be overcome with the use of a baby or silk toothbrush \\
\hline 7. Saline and salt-water rinses are useful, commercial mouthwashes may exacerbate oral discomfort and reduce healing \\
\hline 8. Encourage patients to take adequate oral fluid intake and a well-balanced diet \\
\hline 9. Avoid using alcohol and tobacco \\
\hline 10. Spicy, rough or crunchy foods have the potential to irritate the mucosa or damage the mucosal lining or gums, therefore are better avoided \\
\hline $\begin{array}{l}\text { 11. Dental flossing once a day at night is useful, however, not to be performed in patients with thrombocytopenia, clotting disorder or those receiving } \\
\text { radiotherapy as the severity of OM intensifies. }\end{array}$ \\
\hline 12. Oral sponges may be preferred in patients who find it difficult to use a toothbrush \\
\hline 13. A nurse may assist patients in oral care where patients cannot undertake their own oral hygiene, usually using an irrigating apparatus with saline \\
\hline 14. Some patients may not tolerate sodium lauryl sulphate in toothpastes, therefore suitable alternatives must be sought \\
\hline
\end{tabular}

\section{Adjunctive Therapies}

Dignity therapy is an individualized, brief intervention to facilitate the expression of thoughts, feelings, and memories in terminally ill patients that assists in boosting their dignity, reduces psychological distress, and improves quality of life [33]. Training the caregivers in basic skills including oral hygiene measures will improve the quality of oral health and general quality of life in patients on palliative care [34].

\section{Conclusion}

End of life oral health care presents a significant challenge to the oral healthcare provider. Nutrition plays a vital role in the overall well being of a patient and good oral health compliments nutritional enrichment of the entire body. Caregivers need to be educated along with patients about the importance of maintaining a good oral hygiene and reporting any untoward oral symptoms for immediate care and treatment, so as to prevent complications that may be a long drawn battle in the palliative care patient. Good oral health goes a long way in improving the quality of life in palliatve cancer care.

\section{References}

1. Schenker Y, Arnold R (2017) Toward palliative care for all patients with advanced cancer. JAMA oncology. 3(11): 1459-1460.

2. Wiseman MA (2000) Palliative care dentistry. Gerodontology 17(1): 4951.

3. Venkatasalu MR, Murang ZR, Ramasamy DT, Dhaliwal JS, (2020) Oral health problems among palliative and terminally ill patients: an integrated systematic review. BMC Oral Health 20(1): 1-1.

4. Chen X, Chen H, Douglas C (2013) Dental treatment intensity in frail older adults in the last year of life. J Am Dent Assoc 144: 1234-1242.

5. Fischer DJ, Epstein JB, Yao Y, Wilkie DJ (2014) Oral health conditions affect functional and social activities of terminally ill cancer patients. Support Care Cancer 22: 803-810.
6. Shankar A, Roy S, Bhandari M, Rath GK, Biswas AS, et al. (2017) Current Trends in Management of Oral Mucositis in Cancer Treatment. Asian Pac J Cancer Prev 18(8): 2019-2026.

7. Naidu MU, Ramana GV, Rani PU, Mohan IK, Suman A, et al. (2004) Chemotherapy-induced and/or radiation therapy-induced oral mucositis--complicating the treatment of cancer. Neoplasia 6(5): 423431.

8. Bensinger W, Schubert M, Ang KK, Brizel D, Brown E, et al. (2008) NCCN Task Force Report prevention and management of mucositis in cancer care. J Natl Comp Cancer Netw 6(1): 2-4.

9. Blijlevens N, Sonis S (2007) Palifermin (recombinant keratinocyte growth factor-1): a pleiotropic growth factor with multiple biological activities in preventing chemotherapy- and radiotherapy-induced mucositis. Ann Oncol 18(5): 817-826.

10. Quinn B (2020) UK Oral mucositis in cancer Group. Mouth care guidance and support in cancer and palliative care.

11. Sianturi E, Irawati D (2019) The effectiveness of oral cryotherapy to reduce oral mucositis among cancer patients undergoing chemotherapy: A literature review. International Journal of Nursing and Health Services (IJNHS) 2(2): 102-109.

12. Zadik Yehuda (2019) "Photobiomodulation for the palliation of oral mucositis in cancer patients: the future is here." Future Medicine 15(32): 3647-3649.

13. van der Bogt RD, Vermeulen BD, Reijm AN, Siersema PD, Spaander MC, et al. (2018) Palliation of dysphagia. Best practice \& research Clinical gastroenterology 36: 97-103.

14. Childs DS, Jatoi A (2019) A hunger for hunger: a review of palliative therapies for cancer-associated anorexia. Annals of palliative medicine 8(1): 50.

15. Wiseman M (2017) Palliative Care Dentistry: Focusing on Quality of Life. Compendium of Continuing Education in Dentistry 38(8): 529-534.

16. Dans M, Smith T, Back A, Baker JN, Bauman JR, et al. (2017) NCCN Guidelines Insights: Palliative Care, Version 2.2017. J Natl Compr Canc Netw 15(8): 989-997.

17. Walsh D, Davis M, Ripamonti C, Bruera E, Davies A, et al. (2017) Updated MASCC/ESMO consensus recommendations: Management of nausea and vomiting in advanced cancer. Supportive care in cancer 25(1): 333340 . 
18. Navazesh M (2003) How can oral health care providers determine if patients have dry mouth? Journal of the American Dental Association 134(5): 613-618.

19. Gerdin EW, Einarson S, Jonsson M, Aronsson K, Johansson I, et al. (2006) Impact of dry mouth conditions on oral health-related quality of life in older people. Gerondontology 22(4): 219-226.

20. Hopcraft MS, Tan C (2005) Xerostomia an update for clinicians. Australian Dental Journal 55(3): 238-244.

21. Guggenheimer J, Moore PA (2003) Xerostomia etiology, recognition and treatment. Journal of the American Dental Association 134(1): 61-69.

22. Wiseman M (2006) The Treatment of Oral Problems in the Palliative Patient. J Can Dent Assoc 2006 72(5): 453-458.

23. Barbe AG (2017) Long-term use of the sialogogue medications pilocarpine and cevimeline can reduce xerostomia symptoms and increase salivary flow in head and neck cancer (HNC) survivors after radiotherapy. The Journal of Evidence-Based Dental Practice 17(3): 268270.

24. Iovoli AJ, Ostrowski A, Rivers CI, Hermann GM, Groman A, et al. (2020) Two-Versus Four-Times Weekly Acupuncture-Like Transcutaneous Electrical Nerve Stimulation for Treatment of Radiation-Induced Xerostomia: A Pilot Study. The Journal of Alternative and Complementary Medicine 26(4): 323-328.

25. George, Sarah J (2017) What is the role of acupuncture in the treatment of oncology patients with xerostomia? Australian Journal of Acupuncture and Chinese Medicine 11(1): 4-9.

26. Lagman R, Davis M, LeGrand S, Walsh D, Parala A, et al. (2017) SingleDose Fluconazole Therapy for Oral Thrush in Hospice and Palliative Medicine Patients. American Journal of Hospice and Palliative Medicine, 34(7): 645-649.

ISSN: 2574-1241

DOI: 10.26717/BJSTR.2020.31.005046

Pavan Manohar Patil. Biomed J Sci \& Tech Res

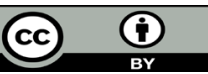

This work is licensed under Creative Commons Attribution 4.0 License

Submission Link: https://biomedres.us/submit-manuscript.php
27. Salins N, Thota RS, Bhatnagar S, Ramanjulu R, Ahmed A, et al. (2019) The Indian Society for Study of Pain, Cancer Pain Special Interest Group guidelines on palliative care aspects in cancer pain management. Indian Journal of Pain 33(4): 49-53.

28. McPherson ML, Walker KA, Davis MP, Bruera E, Reddy A, et al. (2019) Safe and appropriate use of methadone in hospice and palliative care: expert consensus white paper. Journal of pain and symptom management 57(3): 635-645.

29. Delgado MB, Burns L, Quinn C, Moles DR, Kay EJ, et al. (2018) Oral care of palliative care patients-carers' and relatives' experiences. A qualitative study. British Dental Journal 224(11): 881-886

30. Quinn B (2008) Personal Hygiene: Oral Care. In: Dougherty L, Lister $S$ (Eds.), The Royal Marsden Hospital Manual of Clinical Nursing Procedures. Wiley Blackwell, Oxford, ( $7^{\text {th }}$ edn.). pp. 647-659.

31. Eilers J (2004) Nursing interventions and supportive care for the prevention and treatment of oral mucositis associated with cancer treatment. Oncology Nursing Forum 31(4): 13-23.

32. Vissink A, Jansma J, Spijkervet FK, Burlage FR, Coppes RP, et al. (2003) Oral sequelae of head and neck radiotherapy. Critical Reviews in Oral Biology \& Medicine 14(3): 199-212.

33. Xiao J, Chow KM, Liu Y, Chan CW (2019) Effects of dignity therapy on dignity, psychological well-being, and quality of life among palliative care cancer patients: A systematic review and meta-analysis. Psychooncology 28(9): 1791-1802.

34. Kristanti MS, Setiyarini S, Effendy C (2017) Enhancing the quality of life for palliative care cancer patients in Indonesia through family caregivers: a pilot study of basic skills training. BMC palliative care. 16(1): 1-7.

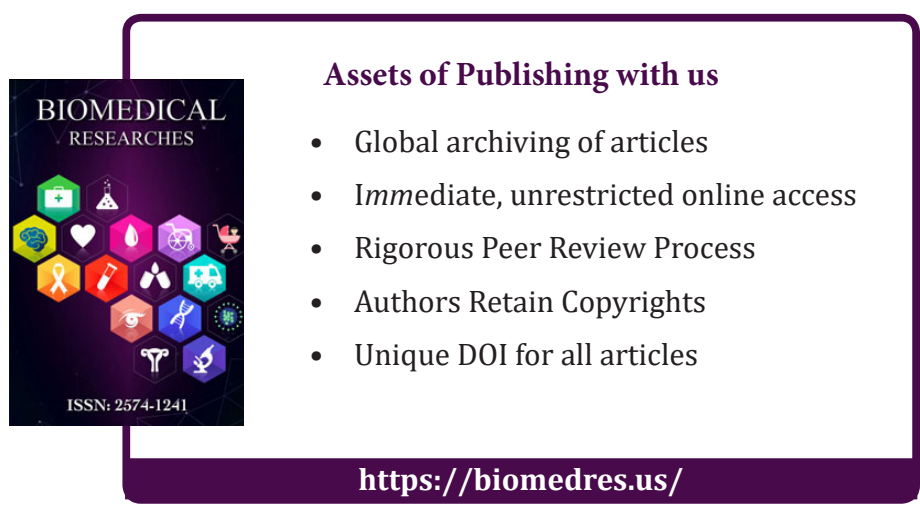

